\title{
Art Relationship between Theme Wall Decoration and Interior Space
}

\author{
Zhao Wenjin \\ Wuchang Institute of Technology \\ Wuhan, 430065 China
}

\begin{abstract}
With the economic development, people's living standard improves, so as to make people's living standard obtain constant improvement. In the contemporary environment, people not only have a usage requirement for living environment, but also have artistic requirement. The interior wall decoration arts works are the ideal furnishing of meeting functional and aesthetic combination.
\end{abstract}

Keywords-theme wall decoration; interior space art;

\section{INTRODUCTION}

Interior space is the indoor place of "cities conducting political, economic, culture and leisure and other public activities", which is the most concentrated and most frequent public activities for people in the city in social life. Indoor public space is one of the modern functional space elements, which is the place that people use every day." With the improvement of people's material life level and constant improvement of living quality, people have practical requirement for living conditions, and it will rise to art demand aspect, and the requirement function and aesthetics achieve the unity.

Theme wall decoration art has a dual nature, on one hand, it exists independently, on the other hand, it needs a certain attachment. The theme wall decoration is an independent existing artistic works, with its unique artistic charm. The expression theme of theme wall decoration is mainly divided into two types: one is tangible themes, such as characters and nature; the other is invisible themes, such as history, culture and social reform.

Theme wall decoration is not only a narrow meaning of "wall decoration", but also integrate "wall" concept into it. The wall surface provides a display foundation for wall decoration, and the wall decoration gives light to wall surface, improving and beautifying the wall surface environment, in order to make wall surface obtain further improvement. Different walls give people different mental feelings, but no matter how to change it, it can use wall decoration texture, color, content advantage to make mental adjustment, so as to make viewers have a comfortable and cheerful feeling. And the true body of interior space is people, which become its creation, communication and resonance objects.

Wall decoration art is a comprehensive application of scientific technology, modern craft technology and modern materials to a certain degree, thus it can fully play its multi-functions of the material itself, integrating the modern and traditional wall decoration feature, adopting multiple artistic techniques, expressing modern life's rich features, achieving the highly unity of function and aesthetics. Wall decoration art is used as interior decoration works, which can beautify people's life in specific space environment, becoming the important part of interior space decoration works.

\section{ThEME WALl DECORATION AND INTERIOR SPACE}

\section{INTERACTION}

The beautifying sense of interior space originates from people's vision, listening and touching sense. For theme wall decoration art, the most essential function and goal are to establish an environment and space suitable for human living. It makes people have self tactile, vision and listening aspect to be pleasant. The visual perception are a main role of interior space cognition, which originates from different simulations of organ for materials of technology and materials, which is different art and material change of material itself. For tactile perception, it is an important gather of wall decoration for cognition, which can make people touch to recognize the thing nature and attribute, such as thing material and types.

Space wall decoration art shows an aesthetic consciousness after materialization, and this consciousness has a certain requirement of consciousness for creators. Most importantly, the filled space shows a pleasant mood function. The theme wall decoration design first surrounds the environment space, and the vision image is absolutely the not mechanical copying perceptual materials, and the similarity is a good grasp for actual life, and this grasp is creative and unique image.

\subsection{Space Environment and Theme Wall Decoration Design}

For decoration, wall is the carrier of presentation, and the two is of close relationship, and the two depend on each other, which is a dialectical relationship between them. From space environment art design to the whole wall decoration art creation process, it has the regulated level. In the creation process, it should conform to its corresponding rules and requirements, according to space environment functions and features to have artistic creation. For wall decoration, the reflected theme spirit and internal aesthetic mood should conform to the whole era development, and it should conform to public aesthetic views in aesthetic taste. Different periods of environment have a great influence on theme wall decoration main body and creative aesthetic trendency. Different regions and environment provide different topics for theme wall decoration creation, producing a strong local feature. And the specific environment has influence on theme wall decoration creation, composition and color.

\subsection{Theme Wall Decoration Artistic Influence on Space Environment}

In the whole space environment, wall decoration is a relative elegant decoration, and also it can conduct gap for space, thus, it can greatly compose the whole environment order. In another aspect, it also shows the artistic charm of the whole space, in order to create an emotional experience atmosphere. Although each new space structure will bring new hope for space use, but the whole space should be composed of system and structure. 
The theme wall decoration has not changed the space wall use situation and use space for function requirement. In addition, from aesthetic view, theme wall decoration will have different aesthetic role in different space locations and environment spaces, so that people will have emotional exchange. Different people have different feelings for space feeling, so in mental and vision aspect, it will make the whole space externals and content have different atmospheres and different artistic appeals.

Andre Pozo created "Holy Aceh Olympic glory" to solve the decoration of church roof and wall gap space. Ma San Aceh church gaint painting---"glory of Aceh Austrian, also known as "St lgnatius of heaven". The Andre Pozogen dome became the extension of the construction, and he has endless walls through the clouds in the picture above, and this is a finite space out of an infinite space, to breakthrough the original limit and the Baroque scenography plays the most incisive. Whether the artistic effect and the church at the level of art or the content of style conforming will leave a deep impression on the art appreciation, for the space, the space is not a box, and the designer is a magician, who tends to view a relatively clear and perfect effect and impression. Thus, the elements of theme wall decoration can closely combine with buck type dynamic penetration and sense of space, in order to achieve the effect of infecting audience.

\section{THEME WALL DECORATION AND INTERIOR SPACE}

\section{DESIGN CREATION}

Theme wall decoration and interior wall artistic conception and style and its adapted coordination relationship complement each other in space environment, which will form a good match in vision, using the conflict and contradiction mode and environment difference to expand the whole space environment range.

\subsection{Theme Wall Decoration Space Design Form}

Theme wall decoration in space environment is limited to specific space formation, with specific belonging objects. The space environment can reflect space composition and design requirement in composition, space style and appearance quality. The so-called space formation is mainly humans or the whole society for multiple demands in the whole space. Thus, the classification process in space science has industrial space, public space and civilian nature of space.

\subsection{Theme Wall Decoration Space Design Structure}

Theme wall decoration has its own features, and it will be restricted by the whole space structure. The so-called space structure mainly refers to that space reflects the specific image and structure. For wall decoration, its works quality depends on works' artistic effect. On the contrary, it should think and research for the role of whole space structure. So, the theme wall decoration location is divided by the whole space to a certain degree, also, it will be restricted by door beam and other structures. The walls restricted by space structure drive theme wall decoration design to conform to various specific structure formations, conforming to space entity surface. And the space structure logics have internal relationships. The theme wall decoration design is composed and fabricated for a specific location of a space, and we should use the laws of wall surface elements, in order to make it not influence the whole effect of wall surface. Also, it can reflect the space structure feature, to make the wall decoration and various space environment designs reflect various light and form emotional world.

\subsection{Theme Wall Decoration Space Environment Art}

Theme wall decoration is used as an element of space art, and it should use unified and harmonious language, and in form and content, it should conform to the whole space style and function, to cope with different demands of space for consideration. So, its creation beginning will be restricted many aspects. When artists write, they should analyze the space function, modeling and environment size aspect in advance, and they should cope with the angle perspective, appreciation distance and lighting, space texture, material texture, resident mentality for overall analysis and research, only by this, we can truly design true space art.

\section{CONCLUSION}

Theme wall decoration can greatly and truly change space environment dimension, but theme wall decoration can conduct remedy for its shortcomings based on actual space demand, eliminating the wall boring. It is a part of space environment, which can form unified decoration style with environment. In addition, to a great extent, it can expand and extend vision feeling in limited range. This art takes human as the center, and this art form can provide spirit and aesthetic resonance for audience. In the era of rapid development, people's life and theme wall decoration works have a great association, and its inheritance should conform to the era development and scientific and technological construction. This is also one of the most important marks of modern theme wall decoration surpassing traditional theme wall decoration art function.

\section{REFERENCES}

[1] Shen Hong, Jiang Zhuqing. Interior public space forged copper gilding decoration. Decoration, 2009(2).

[2] Wang Mogen. Interior wall decoration art color. Film review, 2007(3).

[3] [America]Rudolf Arne Heim. Art and visual perception. Teng Shouyao, Zhu Guan translated. Sichuan: Sichuan People's Press, 2000: 187.

[4] Chen Zhongchen, Li Yanhong. Wall decoration material and design. Liaoning: Liaoning Pictorial Press, 2003.

[5] Yu Meicheng, Tian Weiping, Zhang Daxiang. Mural painting and mural painting creation. Harbin: Heilongjiang Fine Arts Press, 2000. 